\title{
Disentangling the causal inter-relationship between negative life events and depressive symptoms in women: a longitudinal twin study
}

\author{
M. Wichers ${ }^{1 *}$, H. H. Maes ${ }^{2,3}$, N. Jacobs ${ }^{1,4}$, C. Derom ${ }^{5}$, E. Thiery ${ }^{6}$ and K. S. Kendler ${ }^{2}$ \\ ${ }^{1}$ Department of Psychiatry and Neuropsychology, South Limburg Mental Health Research and Teaching Network, EURON, Maastricht \\ University, Maastricht, The Netherlands \\ ${ }^{2}$ Virginia Commonwealth University, Department of Psychiatry and Human and Molecular Genetics, Virginia Institute for Psychiatric and \\ Behavioral Genetics, Richmond, VA, USA \\ ${ }^{3}$ Virginia Commonwealth University, Massey Cancer Center, Richmond, VA, USA \\ ${ }^{4}$ Faculty of Psychology, Open University of the Netherlands, Heerlen, The Netherlands \\ ${ }^{5}$ Department of Human Genetics, University Hospital Gasthuisberg, Katholieke Universiteit Leuven, Belgium \\ ${ }^{6}$ Association for Scientific Research in Multiple Births, Ghent, Belgium
}

Background. Negative life events are strongly associated with the development of depression. However, the etiologic relationship between life events and depression is complex. Evidence suggests that life events can cause depression, and depression increases the risk for life events. Additionally, third factors influencing both phenotypes may be involved. In this work we sought to disentangle these relationships using a genetically informative longitudinal design.

Method. Adult female twins ( $n=536$, including 281 twin pairs) were followed up for measurements of negative life event exposure and depressive symptoms. Four follow-ups were completed, each approximately 3 months apart. Model fitting was carried out using the Mx program.

Results. The best-fitting model included causal paths from life events to depressive symptoms for genetic and shared environmental risk factors, whereas paths from depressive symptoms to life events were apparent for shared environmental factors. Shared latent influence on both phenotypes was found for individual-specific effects.

Conclusions. Life events and depressive symptoms have complex inter-relationships that differ across sources of variance. The results of the model, if replicated, indicate that reducing life event exposure would reduce depressive symptoms and that lowering depressive symptoms would decrease the occurrence of negative life events.

Received 17 August 2010; Revised 8 December 2011; Accepted 15 December 2011

Key words: Depressive symptoms, longitudinal studies, statistical modelling, stressful events, twins.

\section{Introduction}

There is strong accumulated evidence that negative life events play a role in the development of major depression (Kendler et al. 1999, 2001a; Rijsdijk et al. 2001; Paykel, 2003; Hammen, 2005). Interpersonal 'loss' factors, such as bereavement or separation, and other loss experiences such as loss of self-esteem, loss of employment or respected status in the community, or loss of cherished ideas and humiliation (Kendler et al. 2003b), are reported to be potent elicitors of depressive reactions (Brown et al. 1995; Farmer \& McGuffin, 2003). However, the relationship between

* Address for correspondence: Dr M. Wichers, Department of Psychiatry and Psychology, South Limburg Mental Health Research and Teaching Network, EURON, Maastricht University,

Vijverdalseweg 1, Concorde building, Maastricht, The Netherlands.

(Email: m.wichers@sp.unimaas.nl) negative life events and depression is more complex and dynamic than can be accounted for solely by a causal effect of negative life events on depression. Several studies have shown that exposure to negative life events itself is partly under genetic control (Kendler et al. 1993a; Kendler \& Baker, 2007; Vinkhuyzen et al. 2010).

Hammen (1991) introduced the concept of stress generation to describe the finding that people with a history of depression were more likely to expose themselves to negative life events than people without a history of depression (Kendler \& KarkowskiShuman, 1997). The fact that depressed individuals contribute to their experience of stress suggests a causal path from depression to life event exposure. A recent prospective study $(n=826)$ examined the temporal associations between initial chronic stress, neuroticism and follow-up depression severity in a 
sample of out-patients (Brown \& Rosellini, 2011). Evidence was found for both causation (association between initial chronic stress and follow-up depression severity) and the stress generation hypothesis (association between initial depressive severity and follow-up levels of chronic stress). Two other studies, using latent modeling with cross-lagged paths, also found reciprocal associations between major life events and depressive symptoms, supporting the hypotheses of both the causation model and the stress generation model. However, in the latter study reciprocal associations were found only in girls (Ge et al. 1994; Cole et al. 2006). Furthermore, another recent study reported only modest support for 1-year lagged paths from depressive symptoms to major events, and little evidence for paths of the opposite direction (Pettit et al. 2011). Other studies have tried to examine directionality by separating the effects of 'dependent' from 'independent' life events. Dependent life events refer to events that the individual him/herself could have contributed to, such as interpersonal conflicts; independent events refer to those that were not under the control of the individual, such as death of a spouse or a child. Kercher et al. (2009) showed, using path analysis, that not only did depressive symptoms predict later dependent life events but also dependent life events mediated the effects of neuroticism on later depressive symptoms. Other studies (Kendler et al. 1999; Silberg et al. 2001) showed that independent life events predicted future onset of major depression. Thus, previous studies have tried to disentangle the direction of effects and found evidence for bidirectional associations.

However, instead of reciprocal causation, shared causal influence on both life events and depression may also explain the reported phenotypic correlation between negative life events and depression. Potential third factors that are related to both phenotypes are, for example, neuroticism (Kendler et al. 1993b, 2003a; Van Os \& Jones, 1999) or socio-economic status (Brady \& Matthews, 2002; Wang et al. 2010). Neuroticism has been shown phenotypically and genetically to be related to risk for major depression and depressive symptoms (Kendler et al. 1993b; Van Os \& Jones, 1999). In addition, neuroticism is associated with negative life event exposure (Kendler et al. 2003a). As life events are subject to a degree of genetic control, shared genes that influence both life events and depression could be involved. The presence of shared causal influence can be examined using genetically sensitive designs, such as twins studies (see online supplementary material for an explanation of basic behavioral genetic principles and the co-twin control method). The fact that within monozygotic (MZ) twins pairs, matched for both genotype and family environment, life event exposure increased the risk for onset of major depression suggests causal influence of life events on major depression. As this effect was smaller within MZ pairs than dizygotic (DZ) pairs or within the entire population, it was concluded that part of the association (about 1/3) was non-causal and explained by genetic factors that influence both depression liability and exposure to life events. However, in another study that also used the co-twin control method (Middeldorp et al. 2008), it was found that the genes that influenced anxious depression did not overlap with genes influencing life event exposure. One study (Thapar et al. 1998) used structural equation modeling to carry out bivariate genetic analyses of twin data on life events and depressive symptoms. The model supported the presence of shared genetic influences on both phenotypes. However, in this study, the bivariate model was not compared to other models of causal paths from life events to depressive symptoms or the other way around, which may or may not have fitted the data better.

Thus, studies have examined both directionality of effect and potential shared genetic influence, some (Kendler et al. 1999; Silberg et al. 2001; Middeldorp et al. 2008) within the same sample; however, there is disagreement on the role of shared genetic influence. The extent to which shared genes, instead of causal influence of depressive symptoms on new life events, explain the phenotypic association has clinical relevance because stress generation can be hypothesized as a contributing mechanism to the recurrence of depressive episodes (Hammen, 2005). Furthermore, multiple and contrasting pathways of causation may be operating simultaneously depending on the nature of the risk factor. However, no study has yet examined how several sources of variance (additive genetic effects, shared and individual-specific environmental effects) differentially impact on the association between life events and depression.

Therefore, the current longitudinal twin study examined the dynamic within and cross-time associations between negative life events and depressive symptoms using structural equation modeling. To test for directionality of effects and shared sources of influence several models were compared: (i) models with causal paths from life events to depressive symptoms, (ii) models with causal paths from depressive symptoms to life events, and (iii) models without causal paths but with factors for depressive symptoms and life events that were allowed to correlate (which would suggest shared influences). To our knowledge, this is the first longitudinal study using structural equation modeling to disentangle the nature of the dynamic associations between negative life events and depressive symptoms over time. 


\section{Method}

Sample

Subjects $(n=621)$ were taking part in an ongoing, longitudinal, general population twin study on geneenvironment interaction in affective disorders, which has been described in detail elsewhere, and showed a very high degree of compliance with research procedures (Jacobs et al. 2005). The sample consisted of twins $(n=575)$ and siblings of twins $(n=46)$. Following exclusion of those individuals who were non-twin sisters and those with missing data on zygosity, 536 subjects (who were part of 281 twin pairs) had valid life events measurements at the first followup of the study. Given evidence for qualitative differences in the type of environmental stressors that are associated with depression in men and women (Kendler et al. 2001b, 2006) and potential gender differences in the temporal associations between stressors and depressive symptoms (Ge et al. 1994), a female-only sample was chosen to improve homogeneity. The study was approved by the standing ethics committee and subjects provided written informed consent. Zygosity was determined through sequential analysis based on sex, fetal membranes, blood groups and DNA fingerprints (Derom et al. 2006). In 81 pairs, determination of zygosity was based on self and mother's report of standard questions about physical similarity and the degree to which the twins are confused (Spitz et al. 1996; Peeters et al. 1998; Christiansen et al. 2003) and, if necessary, on examination of DNA fingerprints.

\section{Study design}

Subjects were assessed five times at approximately 3to 4-monthly intervals. The average number of days between $\mathrm{T} 0$ and $\mathrm{T} 1$ was 132 , between $\mathrm{T} 1$ and $\mathrm{T} 2 n=91$, between T2 and T3 $n=116$ and between T3 and T4 $n=91$. At T0 assessments were performed at the home of the individuals. For the collection of follow-up data, questionnaires were sent to the participants.

\section{Measurements}

An inventory of recent life events was made based on the event list of the Interview for Recent Life Events (Paykel, 1997). Participants reported on the occurrence of 61 events in the past 6 months (at baseline) and since the last measurement occasion (at follow-up) and rated their impact on a five-point scale (from $1=$ very pleasant to $5=$ very unpleasant). These recent life events were in the domain of 10 categories: work; education; finance; health; bereavement; migration; courtship, marriage and cohabitation; legal, family and social relationships, all representing dateable occurrences involving changes in the external social environment. Events rated as unpleasant (i.e. a score of $4=$ unpleasant or $5=$ very unpleasant) were included in the analysis, and a variable was constructed representing the number of such unpleasant events that had occurred since the last measurement occasion. In the analyses, a negative life event (LE) score was used and coded as follows: $0 \mathrm{LE}=0,1 \mathrm{LE}=1,2$ $\mathrm{LE}=2,3 \mathrm{LE}=3,4 \mathrm{LE}=4, \geqslant 5 \mathrm{LE}=5$, resulting in six categories of life event exposure. As the first (baseline) measurement of negative life events represented occurrences in the past 6 months whereas the follow-up measurements all represented occurrences since the past measurement occasion (approximately 3-month intervals), only the four follow-up measurements were used for the analyses to ensure that all four measurements used the same phenotype.

For the measurements of depressive symptoms, a validated self-report measure was used. At baseline and at each of the four follow-ups, subjects filled in the 90-item Symptom Checklist (SCL-90; Derogatis et al. 1973). The dimension of depressive symptomatology consists of 16 items such as 'feeling low in energy or slowed down', 'feeling no interest in things' or 'experiencing feelings of worthlessness'. Subjects were instructed to rate the degree of discomfort associated with each depressive symptom during the past week on a five-point scale ranging from 'not at all' to 'extremely'. A continuous weighted depression score (sum of scores of the depression items divided by number of items filled in) was calculated at each measurement occasion. Table 1 shows the number of subjects at each time point, the time interval between time points and the average life event and SCL-90 scores at each time point. The mean age of the sample at $\mathrm{T} 1$ was 28 years (range $=18-46$ years). For information on attrition, see supplementary online material. As the measurements of life events and depressive symptoms were analyzed within one model, only the four follow-up measurements of depressive symptoms were used and data were transformed into six categories of symptoms, each with equal numbers of observations (see online material for a further explanation of this choice).

\section{Analyses}

Model fitting was performed using the Mx program (Neale et al. 2003). Several different plausible models that may explain the observed phenotypic association between depressive symptoms and life events were modeled and compared. The models were chosen to reflect the different possibilities of how negative life events and depressive symptoms may be associated 


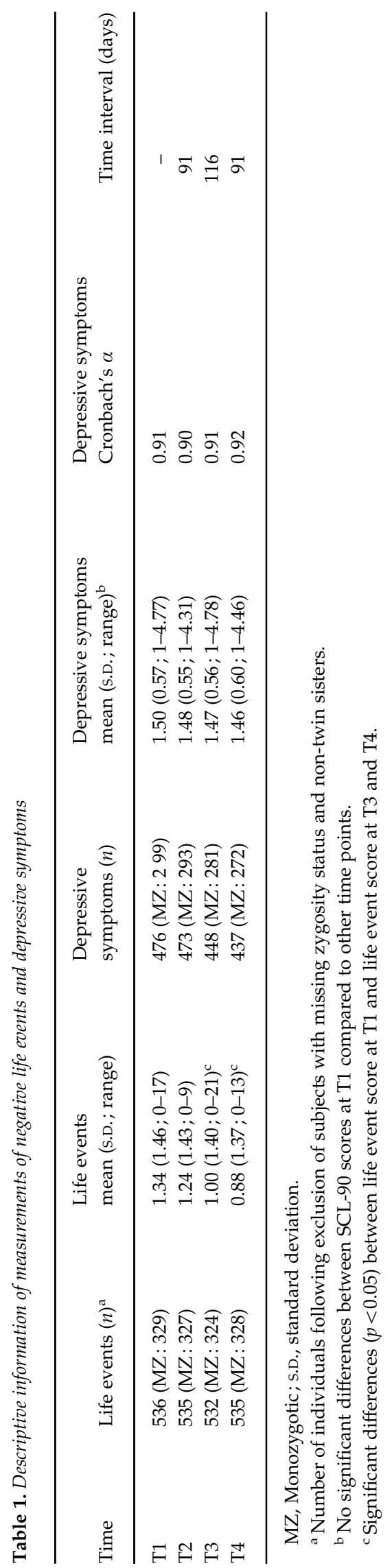

with one another (whether they might be causally affecting each other (bi- or unidirectionally) or whether a common latent construct is affecting both phenotypes simultaneously. Similar types of models have been tested in a previous study on the relationship between peer deviance and conduct disorder (Kendler et al. 2008).

The models are illustrated in Fig. 1 for the genetic paths, but apply equally to shared and individualspecific environmental paths. The first one assumes one latent factor for each phenotype that influences either exposure to life events or experience of depressive symptoms at all time points. This model is called the 'causal factor model' (Fig. 1a). It also includes causal paths between life events and depressive symptoms. Three different causal factor models were tested: (1) a model with bidirectional cross-time causal paths from life events to depressive symptoms (from $\mathrm{LE}_{1}$ to $\mathrm{DS}_{2}$; from $\mathrm{LE}_{2}$ to $\mathrm{DS}_{3}$; from $\mathrm{LE}_{3}$ to $\mathrm{DS}_{4}$ ) and vice versa (from $\mathrm{DS}_{1}$ to $\mathrm{LE}_{2}$; from $\mathrm{DS}_{2}$ to $\mathrm{LE}_{3}$; from $\mathrm{DS}_{3}$ to $\left.\mathrm{LE}_{4}\right)$; (2) a model including only unidirectional paths from life events to depressive symptoms; and (3) a model including only unidirectional paths from depressive symptoms to life events. The latter two models are nested within the first bidirectional model.

The second model (Fig. 1b), which is called the 'simple causal model', assumes that separate independent latent factors influence life events and depressive symptoms at each of the four measurements occasions instead of having one common factor for each phenotype. Three versions of this model were also tested, one with bidirectional paths between the phenotypes and two with either one of the unidirectional paths.

The third model (Fig. 1c) is called the 'correlated factor model'. This model postulates that phenotypic correlations between life events and depression arise from a correlation between the latent factors that influence life events and depression.

Initially, all models include cross-time withinphenotype paths (hereafter 'simplex paths': for example, paths from $\mathrm{T} 1$ to $\mathrm{T} 2, \mathrm{~T} 2$ to $\mathrm{T} 3$ and $\mathrm{T} 3$ to $\mathrm{T} 4$ within both phenotypes). See online material for further details on model characteristics.

Nested models were compared by evaluating decline in fit using $\chi^{2}(\mathrm{df})$ tests. For the evaluation of nonnested models the Bayesian Information Criterion (BIC; Schwartz, 1978) was used. The model with the lowest BIC is considered as the model with the best balance between explanatory power and parsimony. Another frequently used criterion is Akaike's Information Criterion (AIC). The optimum fit as considered by the AIC is typically shifted more towards high explanatory power and less to parsimony as compared to the BIC. The BIC is the criterion used in 
(a)

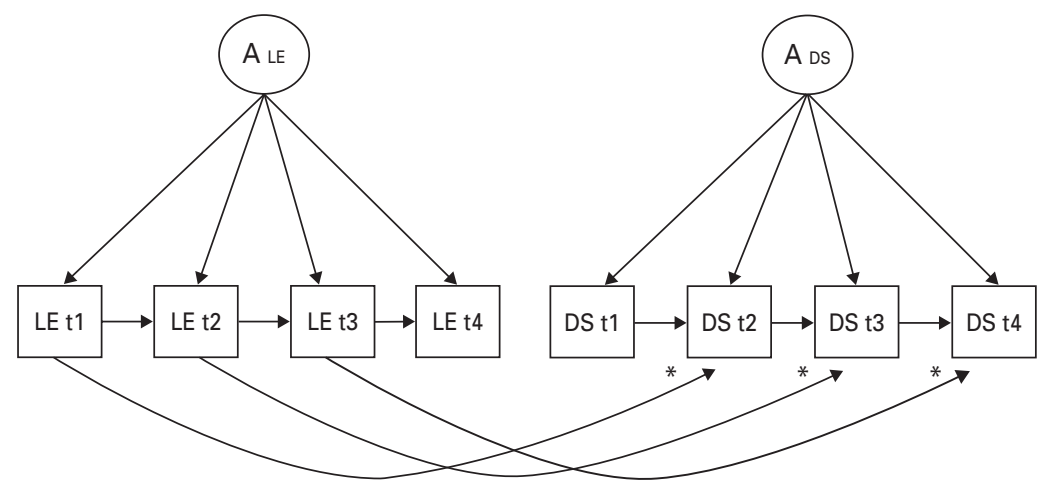

(b)

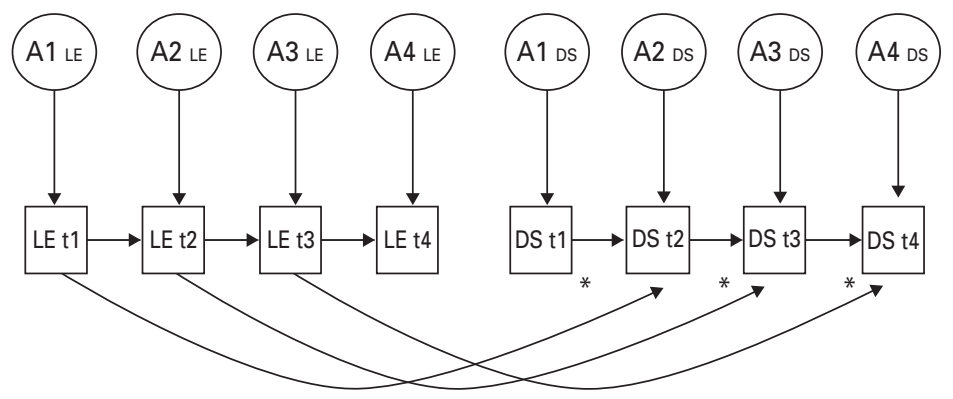

(c)

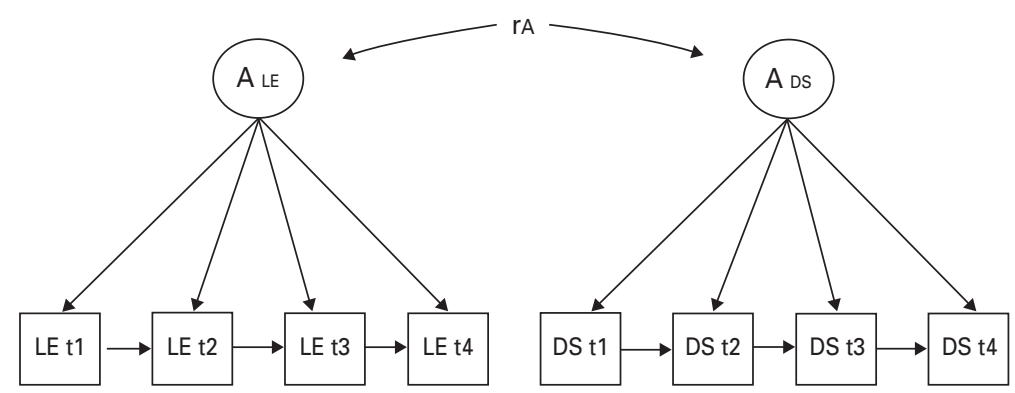

Fig. 1. (a) The causal factor model; (b) the simple causal model; and (c) the correlated factor model. * This model was also tested with the lower arrows pointing in the opposite direction (from depressive symptoms towards life events: DS t 1 to LE $\mathrm{t} 2$, DS t 2 to LE $\mathrm{t} 3$ and DS $\mathrm{t} 3$ to LE $\mathrm{t} 4$ ) and with the lower arrows in both directions simultaneously.

this study because it performs well with such complex models (Markon \& Krueger, 2004). For completeness, however, both the AIC and the BIC values are shown in the description of the results.

First, we fitted a fully saturated model with separate means, variances and covariances, as a baseline for model comparisons. Second, we then tested assumptions of the twin modeling such as equality of means and variances by twin order and zygosity. Third, a triple Cholesky decomposition model for genetic factors (A), shared environmental (C) and individualspecific factors (E) was fitted. This is a saturated model of the observed genetic and environmental variances and covariances (see online material for a further explanation of the Cholesky decomposition and an overview of fit indices for all models tested). We simplified genetic factors first, then shared environmental and then individual-specific factors (see Table 2). The same model testing procedure was followed for each variance component. First, for both the causal factor and simple causal models, the bidirectional models were compared to the unidirectional models to see whether fit would significantly deteriorate after removing one of the directional paths. The best of all causal factor models and the best of all simple causal models were retained. Second, the non-nested bestfitting causal factor, simple causal model and correlated factor model were compared using the BIC. Finally, we tested whether simplex paths could be dropped from the best-fitting model resulting from the 
Table 2. Model testing procedure for variance components $(a) A,(b) C$ and $(c) E$

(a) Variance component A

\begin{tabular}{|c|c|c|c|c|c|}
\hline (a) Variance component A & Model & & & & \\
\hline & Description & $-2 \mathrm{LL}$ & $\mathrm{df}$ & $-2 \mathrm{LL}$ change $(\mathrm{df})$ & Result $^{\mathrm{a}}$ \\
\hline
\end{tabular}

Causal factor: nested model evaluation

Variance component A: bi- and unidirectional causal factor models
$\mathrm{LE} \leftrightarrow \mathrm{DS}$
$10934.698 \quad 3874 \quad-$

II LE $\rightarrow$ DS $\quad 10935.432 \quad 3875 \quad 0.73(1) \quad \leftarrow$ can be dropped

III LE $\leftarrow$ DS $\quad 10949.188 \quad 3875 \quad 14.49(1) \quad \rightarrow$ cannot be dropped

Simple causal: nested model evaluation

Variance component A: bi- and unidirectional simple causal models

IV LE DS $\quad 10936.614 \quad 3874 \quad-\quad$ -

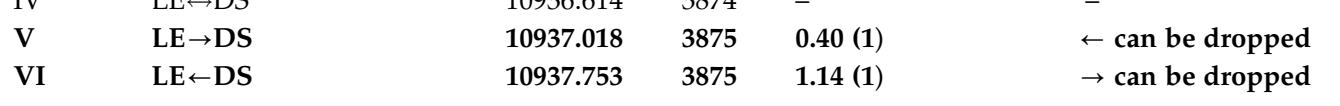

Comparison of non-nested best models using BIC

Variance component A: comparison non-nested best models

\begin{tabular}{lllll}
\hline Description & $-2 \mathrm{LL}$ & df & AIC & BIC \\
\hline CF $:$ LE $\rightarrow$ DS & $\mathbf{1 0 9 3 5 . 4 3 2}$ & $\mathbf{3 8 7 5}$ & $\mathbf{3 1 8 5 . 4 3 2}$ & $\mathbf{- 5 4 6 3 . 4 7 9}$ \\
SC: LE $\rightarrow$ DS & 10937.018 & 3875 & 3187.018 & -5462.686 \\
SC: LE $\leftarrow$ DS & 10937.753 & 3875 & 3187.753 & -5462.318 \\
Corr. factor & 10939.443 & 3875 & 3189.443 & -5461.473
\end{tabular}

Variance component A: comparison simplex paths of best-fitting model

Simplex paths: nested model evaluation of best-fitting model

\begin{tabular}{|c|c|c|c|c|}
\hline Description & $-2 \mathrm{LL}$ & $\mathrm{df}$ & $-2 \mathrm{LL}$ change $(\mathrm{df})$ & Result \\
\hline Best model CF: LE $\rightarrow$ DS & 10935.432 & 3875 & - & - \\
\hline Drop of simplex paths & 10935.515 & 3877 & $0.08(2)$ & $\begin{array}{l}\text { Simplex paths can be } \\
\text { dropped }\end{array}$ \\
\hline Description & $-2 \mathrm{LL}$ & $\mathrm{df}$ & AIC & BIC \\
\hline $\begin{array}{l}\text { CF: } L E \rightarrow D S \\
\text { Drop of simplex paths }\end{array}$ & 10935.515 & 3877 & 3181.515 & -5469.080 \\
\hline
\end{tabular}

Best model for A

Drop of simplex paths 
(b) Variance component C using best model for A (IIc) Model

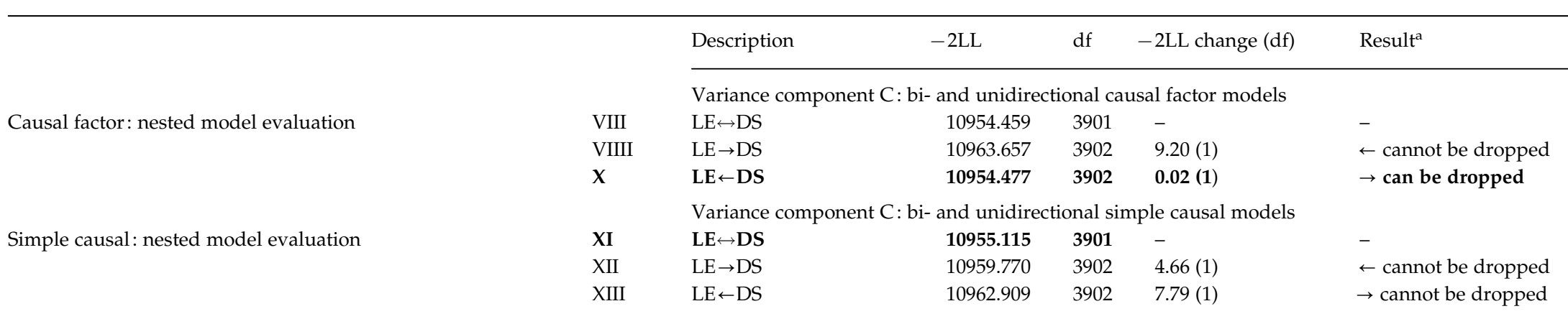

Comparison of non-nested best models using BIC criterion

Variance component C: comparison non-nested best models

\begin{tabular}{lllll}
\hline Description & -2 LL & df & AIC & BIC \\
\hline CF $:$ LE $\leftarrow$ DS & $\mathbf{1 0 9 5 4 . 4 7 7}$ & $\mathbf{3 9 0 2}$ & $\mathbf{3 1 5 0 . 4 7 7}$ & $\mathbf{- 5 5 3 0 . 1 2 2}$ \\
SC: LE $\leftrightarrow$ DS & 10955.115 & 3901 & 3153.115 & -5526.982 \\
Corr. factor & 10964.009 & 3902 & 3160.009 & -5525.356
\end{tabular}

Variance component C: comparison simplex paths of best-fitting model

Simplex paths: nested model evaluation of best-fitting model

\begin{tabular}{|c|c|c|c|c|}
\hline Description & $-2 L L$ & df & $-2 \mathrm{LL}$ change $(\mathrm{df})$ & Result $^{\mathrm{a}}$ \\
\hline Best model CF: LE $\leftarrow$ DS & 10954.477 & 3902 & - & - \\
\hline Drop of simplex paths & 10957.498 & 3904 & $3.02(2)$ & Simplex paths can be dropped \\
\hline Description & $-2 \mathrm{LL}$ & $\mathrm{df}$ & AIC & $\mathrm{BIC}$ \\
\hline $\begin{array}{l}C F: L E \leftarrow D S \\
\text { Drop of simplex paths }\end{array}$ & 10957.498 & 3904 & 3149.498 & -5534.253 \\
\hline
\end{tabular}

Best model for C
(c) Variance component E using best model for A and C (Xc)

Model

Description

$-2 \mathrm{LL}$

df $\quad-2$ LL change (df ) Result ${ }^{\mathrm{a}}$

Causal factor: nested model evaluation

\begin{tabular}{llllll}
\multicolumn{7}{c}{ Variance component E: bi- and unidirectional causal factor model } \\
XV & LE $\leftrightarrow$ DS & 11040.907 & 3920 & - & - \\
XVI & LE $\rightarrow$ DS & 11053.902 & 3921 & $12.995(1)$ & $\leftarrow$ cannot be dropped \\
XVII & LE $\leftarrow$ DS & $\mathbf{1 1 0 4 3 . 1 0 2}$ & $\mathbf{3 9 2 1}$ & $\mathbf{2 . 1 9 5 ( 1 )}$ & $\rightarrow$ can be dropped
\end{tabular}


(c) Variance component E using best model for A and C (Xc) Model

Simple causal: nested model evaluation

Comparison of non-nested best models using BIC

Simplex paths: nested model evaluation of best-fitting model

Variance component E: bi- and unidirectional simple causal models

$\begin{array}{llllll}\text { XVIII } & \text { LE } \leftrightarrow \text { DS } & \mathbf{1 1 0 9 1 . 9 7 1} & 3928 & - & - \\ \text { XIX } & \text { LE } \rightarrow \text { DS } & 11099.040 & 3929 & 7.069(1) & \rightarrow \text { cannot be dropped } \\ \text { XX } & \text { LE } \leftarrow \text { DS } & 11101.807 & 3929 & 9.836(1) & \rightarrow \text { cannot be dropped }\end{array}$

Variance component E: comparison non-nested best models

\begin{tabular}{llllll}
\cline { 2 - 5 } & Description & -2 LL & df & AIC & BIC \\
\cline { 2 - 6 } XVII & CF: LE↔DS & 11043.102 & 3921 & 3201.102 & -5539.408 \\
XVIII & SC: LE↔DS & 11091.971 & 3928 & 3235.971 & -5534.720 \\
XXI & Corr. factor & $\mathbf{1 0 9 9 8 . 7 4 7}$ & $\mathbf{3 9 2 1}$ & $\mathbf{3 1 5 6 . 7 4 7}$ & $\mathbf{- 5 5 6 1 . 5 8 5}$
\end{tabular}

Variance component E: comparison simplex paths of best-fitting model

Description $\quad-2 L L \quad$ df $\quad-2 L L$ and df change Result*

\begin{tabular}{llllll}
\cline { 2 - 5 } XXI & Best model: corr. factor & $\mathbf{1 0 9 9 8 . 7 4 7}$ & 3921 & - & - \\
XXIc & E Simplex paths & 11007.896 & 3923 & $9.149(2)$ & $\begin{array}{l}\text { Simplex paths cannot } \\
\text { be dropped }\end{array}$ \\
\cline { 2 - 6 } & Description & $-2 \mathrm{LL}$ & df & AIC & BIC \\
\cline { 2 - 6 } & Corr. factor & $\mathbf{1 0 9 9 8 . 7 4 7}$ & 3921 & $\mathbf{3 1 5 6 . 7 4 7}$ & $\mathbf{- 5 5 6 1 . 5 8 5}$ \\
\hline
\end{tabular}

\section{Best model for $\mathrm{E}$}

A, Additive genetic effects; C, shared environmental effects; E, individual-specific effects; -2LL, -2 log likelihood ; df, degrees of freedom; AIC, Akaike Information Criterion; BIC, Bayesian Information Criterion; LE, life events; DS, depressive symptoms; Corr. factor, correlated factor model; CF, causal factor model; SC, simple causal model.

First, nested models were evaluated by the decline in fit $(-2 \mathrm{LL})$ in relation to $\mathrm{df}$ using $\chi^{2}$ tests. In a second step, the best models resulting from these evaluations were compared to other non-nested models and evaluated using the BIC. In a third step, the best of these models was selected and we tested whether simplex paths could be dropped, again by evaluating the decline in fit using $\chi^{2}$ tests. The best-fitting A model was used for further testing of the C model and, similarly, the best resulting C model was used for further testing of the E model (see online material for additional information). Note that we may repeat the statistics of the models for different comparisons; however, the model number reflects this.

${ }^{\text {a } C r i t i c a l ~} \chi^{2}$ values are $3.84(\mathrm{df}=1)$ and $5.99(\mathrm{df}=2)$. Above the critical values there is a significant deterioration of fit.

Best-fitting models are marked in bold. 
Table 3. Within- and cross-time cross-phenotype correlations

\begin{tabular}{lllll}
\hline & $\begin{array}{l}\text { Life } \\
\text { events t1 }\end{array}$ & $\begin{array}{l}\text { Life } \\
\text { events t2 }\end{array}$ & $\begin{array}{l}\text { Life } \\
\text { events t3 }\end{array}$ & $\begin{array}{l}\text { Life } \\
\text { events t4 }\end{array}$ \\
\hline Depressive symptoms t1 & 0.37 & $\mathbf{0 . 2 7}$ & $\mathbf{0 . 2 4}$ & $\mathbf{0 . 1 8}$ \\
Depressive symptoms t2 & 0.22 & 0.30 & $\mathbf{0 . 2 4}$ & $\mathbf{0 . 2 0}$ \\
Depressive symptoms t3 & 0.26 & 0.27 & 0.33 & $\mathbf{0 . 2 8}$ \\
Depressive symptoms t4 & 0.24 & 0.23 & 0.30 & 0.32 \\
\hline
\end{tabular}

Numbers in bold represent correlations between depressive symptoms and life events later in time. Numbers in italics represents correlations between life events and depressive symptoms later in time. Diagonals represent within-time cross-phenotype correlations.

latter evaluation. The final model was then used in further testing of other ( $\mathrm{C}$ and $\mathrm{E}$ ) variance components. Because directional effects were not hypothesized to be different across time points, the simplex paths in addition to the causal paths were $a$ priori set to the same value.

As temporal changes in variance in longitudinal studies with repeated measurements are informative about the underlying developmental process (Eaves et al. 1986), path coefficients of the model on the first occasion only were standardized so that the phenotypic variance is unity. Variances at subsequent occasions were expressed relative to their initial values. Therefore, the path coefficients can exceed unity, particularly when variances are increasing over time.

\section{Results}

\section{Phenotypic correlations}

Table 3 shows the within- and across-time phenotypic correlations between negative life events and depressive symptoms. Correlations below the diagonal depict the prediction of depressive symptoms by previously experienced life events. Those above the diagonal are informative for the prediction of life event exposure by prior depressive symptoms. These two sets of correlations are broadly similar in magnitude, suggesting that causal effects are probably operating in both directions. However, the hypothesis that a third factor influences both phenotypes is also consistent with this pattern of correlations.

\section{Model fitting}

First, fully saturated models were fitted on the observed variables. The model in which means and variances were equated across groups (twin 1, twin 2, MZ and DZ twins) did not fit significantly worse than the fully saturated model as evaluated by the BIC. Second, a Cholesky model with equal means and variances across groups but with different thresholds per time point was tested and compared to the same model but with equal thresholds across time. The latter fitted best and was used for further model testing (for information on fit statistics of these models see online material). Table 2 summarizes the results from the correlational and causal models tested (see online material for further information on the model testing procedure).

For the genetic factor (A), the causal factor model with paths going from negative life events to depressive symptoms (model II) provided the best fit. In addition, simplex paths could be dropped from this model without deterioration in fit. It should be noted that the nested model evaluation of the simple causal models could not distinguish the two unidirectional models. Both unidirectional models were preferred over the bidirectional model. The differences in BIC between model II on the one hand and models $\mathrm{V}$ and VI on the other were small (Table $2 a$ ). For the shared environmental factor $(\mathrm{C})$, model $\mathrm{X}$ was the best fit. This model also had a causal factor structure, with one latent factor per phenotype influencing observations at all time points. In contrast to the previous model, this one included unidirectional causal paths from depressive symptoms to life events. Also here, simplex paths could be dropped without fit deterioration (Table $2 b$ ). Finally, the individual-specific factor (E) was simplified. The best-fitting model for the E factor was the correlated factor model (model XXI). Simplex paths could not be dropped from this model without significant deterioration in fit (Table $2 c$ ).

\section{Best-fit model}

The overall best-fit model (Fig. 2) had the following four key features. First, the genetic risk factors for negative life events and depressive symptoms could be best understood as two single common factors, so 

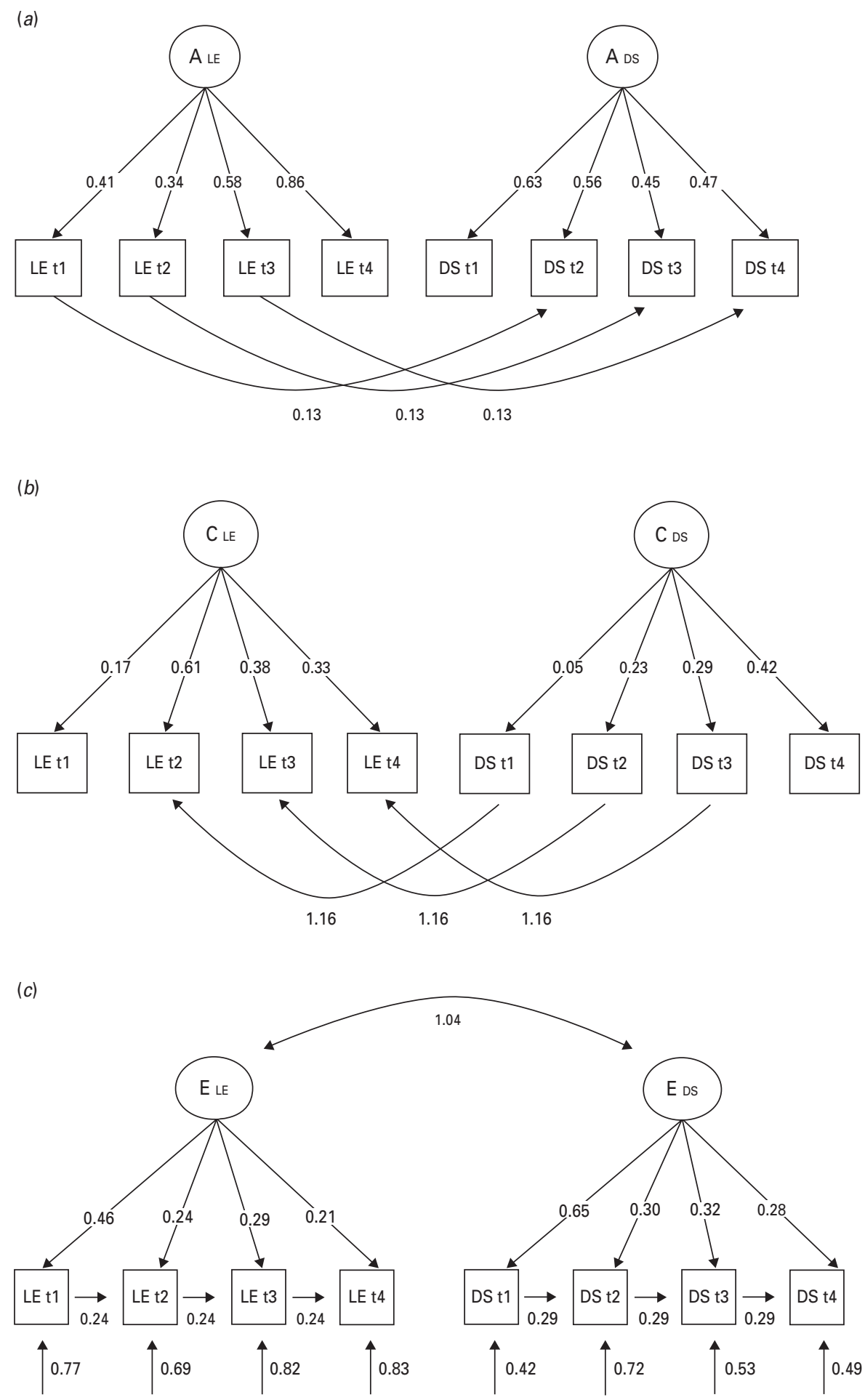

Fig. 2. Resulting best-fit model of the additive genetic (A), shared environmental (B) and individual-specific (E) influences on negative life event exposure and depressive symptoms. (a) The causal factor model with paths from negative life events to depressive symptoms (see Table $2 a$ ). (b) The causal factor model with paths from depressive symptoms to negative life events (see Table 2b). (c) The correlated factor model with simplex paths and additional observation-specific effects (see Table $2 c$ ).

that the same genetic factors influenced the phenotypes at all four time points (Fig. 2a). Second, the causal paths in the genetic portion of the model went from negative life events to depression. Third, the shared environmental factors showed a similar structure to that seen for genetic factors, but with causal 
Table 4. Variance components for life events and depressive symptoms at all time points

Percentage explained variance for A, C and E

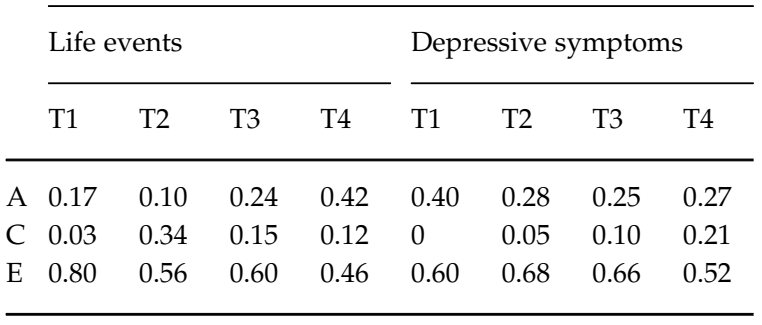

A, Additive genetic effects; $C$, shared environmental effects; $\mathrm{E}$, individual-specific effects.

paths going in the opposite direction, that is from depressive symptoms to life events (Fig. $2 b$ ). Fourth, by contrast, the individual-specific environmental influences on life events and depressive symptoms could best be modeled as two correlated latent factors (Fig. 2c). In addition, forward transmission was present in the $\mathrm{E}$ model for both negative life events and depressive symptoms from $\mathrm{T} 1$ to $\mathrm{T} 2, \mathrm{~T} 2$ to $\mathrm{T} 3$ and $\mathrm{T} 3$ to T4. That is, levels of life event exposure at one time point had a direct impact on levels of life event exposure at the next time point. Furthermore, depressive symptoms at one time period directly impacted on depressive symptoms at the next time point.

Table 4 shows the estimates for $\mathrm{a}^{2}, \mathrm{c}^{2}$ and $\mathrm{e}^{2}$ for negative life events and depressive symptoms at all time points, obtained from the best-fit model (model XXI).

\section{Discussion}

\section{Findings}

This study sought to clarify the causal relationship between exposure to negative life events and depressive symptoms using a longitudinal genetically informative design. The most striking features of the best-fitting model are the following. Life events and depressive symptoms had a complex interrelationship that differed depending on the source of variance considered. The best-fitting model was a combination of model specifications with directional paths across time between the two phenotypes and with a correlated factor structure, implying shared influences on both phenotypes. Thus, both causal paths and shared influences explained the phenotypic correlations between negative life events and depressive symptoms. Furthermore, causal paths between the two phenotypes went in both directions, depending on the source of variance. Genetic factors impacted on exposure to life events, which in turn influenced the risk for depressive symptoms. Thus, although the exposure to life events itself was influenced by genetic factors, the life events were causal to the development of depressive symptoms. This model thus shows two different paths by which genes may influence depressive symptoms. First, there is the direct path of additive genetic influences on depressive symptoms. These are probably genes that affect people's vulnerability to depression by acting on biological, cognitive or psychological processes, for example by influencing people's affective processing or genes associated with increased stress responses to negative situations (Wichers et al. 2007, 2009). These genes thus act 'inside the skin'. Second, there is an indirect path from genes to depressive symptoms via exposure to negative life events. These genes act on depression by creating an environment (outside the skin) that exposes the individual to negative life events. Genes that influence the ability to decide on the important choices in life (e.g. choosing one's marital partner, study, job) may impact on exposure to life events. Moreover, genetic influences on having low emotional intelligence or a difficult personality (e.g. high levels of neuroticism) may set people up for the loss of relationships, friendships or jobs. The indirect path is a typical example of active geneenvironment correlation (Plomin et al. 1977). According to the current model, however, it can be calculated from the path coefficients and the total variance that only around $1-2 \%$ of the variance at each time point was explained by indirect (outside the skin) paths and $98-99 \%$ by the direct (inside the skin) pathway (exact numbers of standardized total effects of the direct and indirect genetic paths available upon request). However, this does not mean that the indirect pathway is non-existent. This model examined effects across time. It is possible that the effects of this path may be larger when examining the effects of life events on depressive symptoms within time, examining the effects of life events of the past 3 months, instead of the life events as reported one time point before.

For shared environmental influences the coefficients of the latent factor on depressive symptoms were modest; however, the strength of the causal path from depressive symptoms to life events was fairly large (see Fig. 2). An example of such shared environmental influences is parental divorce or shared adverse upbringing leading to adult depressive symptoms. The expression of these depressive symptoms may then further increase the risk for life events, such as having a divorce or having relational problems themselves. Thus, also here there is a direct path to the experience of negative life events and an indirect path via the experience of depressive symptoms. The indirect 
paths (from DS T2 to LE T3 and from DS T3 to LE T4) contribute meaningful percentages of the total effect of the shared environment, $31 \%$ and $57 \%$ respectively. This finding is consistent with Hammen's hypothesis of stress generation and the idea that the relationship between life events and depressive symptoms is bidirectional (Hammen, 1991, 2005). The fact that experience of depressive symptoms themselves selects an environment of increased stress exposure would imply that lowering depressive symptoms also positively impacts on the environment people create around themselves (Hammen, 1991). This finding also emphasizes the need to resolve residual symptoms following a depressive episode to prevent recurrence (Kennedy \& Paykel, 2004).

For individual-specific effects the correlated factor structure fitted best. Thus, phenotypic correlations between life events and depression arise partly from a correlation between the $\mathrm{E}$ latent factors that influence life events and depression. Shared individual-specific effects are both affecting risk for life events and depressive symptoms. These could involve experiences such as a physical illness, simultaneously leading to the loss of a job and to feeling down. Another example is being bullied in childhood, leading both to an altered way of coping with daily life situations, resulting in an increased level of life events, and to a more active stress system, resulting in mood symptoms.

\section{Causal paths versus shared causal influence}

The finding that genes impact on depressive symptoms through their effect on exposure to negative life events is in agreement with previous studies. Kendler \& Karkowski-Shuman (1997) concluded that genetic risk factors for major depressive disorder increase the probability of experiencing stressful life events. That study, however, mentioned the possibility that not only causal effects from life events to depression but also shared causal influence on both phenotypes might have explained the findings. Neuroticism seems a likely candidate because effects of neuroticism on both phenotypes have been shown (Kendler et al. $1993 b, 2003 a$ ) and neuroticism is partially heritable (Viken et al. 1994; Jang et al. 1996). However, a study using path analyses (Kercher et al. 2009) showed that the best-fitting model did not include any direct paths from neuroticism to depression. Instead it included paths from neuroticism to dependent negative life events and negative thoughts, which in turn, had causal paths to depression. These findings are consistent with those of the current study, in which the best-fitting model showed genetic effects on negative life events, which in turn had causal paths to depressive symptoms.
The current model thus suggests that shared genetic and environmental influences on negative life events and depressive symptoms are expressed at the phenotypic level (at the level of observed negative life events that cause depressive symptoms) and not at the latent level (the level of the latent genetic factor that has direct paths to both negative life events and depressive symptoms). The fact that the current design is able to distinguish models favoring shared influences expressed at the phenotypic level from those favoring shared genetic influence at the latent level is not a trivial or purely theoretical advantage. These two interpretations have substantially different implications for the prevention of depressive symptoms or negative life events. If shared genes indeed impact on depressive symptoms at the phenotypic level, through the generation of negative life events, it follows that decreasing life event exposure should decrease the risk for depressive symptoms, whereas this would not be the case when shared genes exert their effects at the latent level. Likewise, it follows that decreasing the level of depressive symptoms should decrease the risk for negative life events. The current results revealed a fairly large effect of the indirect path to life events, but small effects of the indirect path to depressive symptoms. Because of the clinical relevance of the outcome, there is an urgent need for replication and further examination of these effects.

A drawback of the current model, however, is that it leaves little room for the effect of independent negative life events on depressive symptoms. Previous work has clearly shown that fateful negative events that are uncontrollable by people themselves impact on the risk for depression (Kendler et al. 1999, 2000). The model, therefore, must be interpreted with caution. Moreover, explanations for the lack of effects of independent life events were investigated further. When life events were split into dependent and independent life events, regression analyses showed that more items in the list of life events were dependent (marital discord, fights and arguments with friends or family, loss of job, etc.) than independent (death of partner or close relatives, serious illness, partner or close relatives with serious illness, etc.). In addition, the dependent life events were far more frequent than the independent ones, and the dependent life events had a much stronger depressogenic influence. Therefore, it may be that dependent life events dominated the impact of independent life events in the process of model fit comparisons. Separate analyses for independent and dependent life events might have given a different picture for the associations between independent life events and depressive symptoms, with stronger causal paths of purely environmentally influenced life events on depressive symptoms. 
However, because independent life events were rare, such an analysis would be likely to suffer from a lack of power. Dependent life events are usually more dominant in younger life. The mean age of the twins was 28 years, which is relatively young, and which might explain the high frequency of dependent compared to independent life events in this sample.

\section{Methodological issues}

Models II, V and VI, in the specification of the A variance component, showed only very small differences in BIC values $(\triangle B I C<2)$. Therefore, caution is warranted regarding the resulting model. The study may have lacked the power to differentiate well between these models. Although not small, the current sample $(n=536)$ is smaller than the sample size of a previous study $(n=1492)$ that used similar model-fitting analyses (Kendler et al. 2008). The models, however, in the specification of the $C$ and $E$ variance component could be differentiated with sufficient confidence (Raftery, 1995). In addition, the measurements rely on retrospective self-report of negative life events and depressive symptoms. Finally, this was a female sample only. Therefore, the results may not be generalizable to men.

To summarize, the current study suggests that both reciprocal causation, using cross-time intervals of approximately 3 months, and shared latent influences explained the inter-relationship between negative life events and depressive symptoms. The results of our study should be interpreted with caution as these questions need to be addressed by further studies and replicated before they deserve wide acceptance.

\section{Note}

Supplementary material accompanies this paper on the Journal's website (http:/ /journals.cambridge.org/ psm).

\section{Acknowledgements}

This research was supported by the Dutch Organization for Scientific Research; the Fund for Scientific Research, Flanders and Twins, a non-profit association for scientific research in multiple births (Belgium) (to the East Flanders Prospective Survey); and the Dutch Medical Council (VENI grant no. 916.76.147) (to Dr M. Wichers). We thank all twins for their cooperation.

\section{Declaration of Interest}

None.

\section{References}

Brady SS, Matthews KA (2002). The influence of socioeconomic status and ethnicity on adolescents' exposure to stressful life events. Journal of Pediatric Psychology 27, 575-583.

Brown GW, Harris TO, Hepworth C (1995). Loss, humiliation and entrapment among women developing depression: a patient and non-patient comparison. Psychological Medicine 25, 7-21.

Brown TA, Rosellini AJ (2011). The direct and interactive effects of neuroticism and life stress on the severity and longitudinal course of depressive symptoms. Journal of Abnormal Psychology 120, 844-856.

Christiansen L, Frederiksen H, Schousboe K, Skytthe A, von Wurmb-Schwark N, Christensen K, Kyvik K (2003). Age- and sex-differences in the validity of questionnairebased zygosity in twins. Twin Research 6, 275-278.

Cole DA, Nolen-Hoeksema S, Girgus J, Paul G (2006). Stress exposure and stress generation in child and adolescent depression: a latent trait-state-error approach to longitudinal analyses. Journal of Abnormal Psychology 115, 40-51.

Derogatis LR, Lipman RS, Covi L (1973). SCL-90: an outpatient psychiatric rating scale - preliminary report. Psychopharmacology Bulletin 9, 13-28.

Derom CA, Vlietinck RF, Thiery EW, Leroy FO, Fryns JP, Derom RM (2006). The East Flanders Prospective Twin Survey (EFPTS). Twin Research and Human Genetics $\mathbf{9}$, 733-738.

Eaves LJ, Long J, Heath AC (1986). A theory of developmental change in quantitative phenotypes applied to cognitive development. Behavior Genetics 16, 143-162.

Farmer AE, McGuffin P (2003). Humiliation, loss and other types of life events and difficulties: a comparison of depressed subjects, healthy controls and their siblings. Psychological Medicine 33, 1169-1175.

Ge X, Lorenz F, Conger R, Elder GH, Simons RL (1994). Trajectories of stressful life events and depressive symptoms during adolescence. Developmental Psychology 30, 467-483.

Hammen C (1991). Generation of stress in the course of unipolar depression. Journal of Abnormal Psychology 100, $555-561$.

Hammen C (2005). Stress and depression. Annual Review of Clinical Psychology 1, 293-319.

Jacobs N, Nicolson NA, Derom C, Delespaul P, van Os J, Myin-Germeys I (2005). Electronic monitoring of salivary cortisol sampling compliance in daily life. Life Sciences 76, 2431-2443.

Jang KL, Livesley WJ, Vernon PA (1996). Heritability of the big five personality dimensions and their facets: a twin study. Journal of Personality 64, 577-591.

Kendler KS, Baker JH (2007). Genetic influences on measures of the environment: a systematic review. Psychological Medicine 37, 615-626.

Kendler KS, Gardner CO, Prescott CA (2003a). Personality and the experience of environmental adversity. Psychological Medicine 33, 1193-1202. 
Kendler KS, Gatz M, Gardner CO, Pedersen NL (2006). A Swedish national twin study of lifetime major depression. American Journal of Psychiatry 163, 109-114.

Kendler KS, Hettema JM, Butera F, Gardner CO, Prescott CA $(2003 b)$. Life event dimensions of loss, humiliation, entrapment, and danger in the prediction of onsets of major depression and generalized anxiety. Archives of General Psychiatry 60, 789-796.

Kendler KS, Jacobson K, Myers JM, Eaves LJ (2008). A genetically informative developmental study of the relationship between conduct disorder and peer deviance in males. Psychological Medicine 38, 1001-1011.

Kendler KS, Karkowski-Shuman L (1997). Stressful life events and genetic liability to major depression: genetic control of exposure to the environment? Psychological Medicine 27, 539-547.

Kendler KS, Karkowski LM, Prescott CA (1999). Causal relationship between stressful life events and the onset of major depression. American Journal of Psychiatry 156, 837-841.

Kendler KS, Neale M, Kessler R, Heath A, Eaves L (1993a). A twin study of recent life events and difficulties. Archives of General Psychiatry 50, 789-796.

Kendler KS, Neale MC, Kessler RC, Heath AC, Eaves LJ (1993b). A longitudinal twin study of personality and major depression in women. Archives of General Psychiatry 50, 853-862.

Kendler KS, Thornton LM, Gardner CO (2000). Stressful life events and previous episodes in the etiology of major depression in women: an evaluation of the 'kindling' hypothesis. American Journal of Psychiatry 157, 1243-1251.

Kendler KS, Thornton LM, Gardner CO (2001a). Genetic risk, number of previous depressive episodes, and stressful life events in predicting onset of major depression. American Journal of Psychiatry 158, 582-556.

Kendler KS, Thornton LM, Prescott CA (2001b). Gender differences in the rates of exposure to stressful life events and sensitivity to their depressogenic effects. American Journal of Psychiatry 158, 587-593.

Kennedy N, Paykel ES (2004). Residual symptoms at remission from depression: impact on long-term outcome. Journal of Affective Disorders 80, 135-144.

Kercher AJ, Rapee RM, Schniering CA (2009). Neuroticism, life events and negative thoughts in the development of depression in adolescent girls. Journal of Abnormal Child Psychology 37, 903-915.

Markon KE, Krueger RF (2004). An empirical comparison of information-theoretic selection criteria for multivariate behavior genetic models. Behavior Genetics 34, 593-610.

Middeldorp CM, Cath DC, Beem AL, Willemsen G, Boomsma DI (2008). Life events, anxious depression and personality: a prospective and genetic study. Psychological Medicine 38, 1557-1565.

Neale MC, Boker SM, Xie G, Maes H (2003). Mx: Statistical Modeling. Department of Psychiatry, Virginia Commonwealth University Medical School, Box 980126: Richmond, VA 23298.

Paykel ES (1997). The Interview for Recent Life Events. Psychological Medicine 27, 301-310.
Paykel ES (2003). Life events and affective disorders. Acta Psychiatrica Scandinavica 108 (Suppl. 418), 61-66.

Peeters H, Van Gestel S, Vlietinck R, Derom C, Derom R (1998). Validation of a telephone zygosity questionnaire in twins of known zygosity. Behavior Genetics 28, 159-163.

Pettit JW, Lewinsohn PM, Seeley JR, Roberts RE, Yaroslavsky I (2011). Developmental relations between depressive symptoms, minor hassles, and major events from adolescence through age 30 years. Journal of Abnormal Psychology 119, 811-824.

Plomin R, DeFries JC, Loehlin JC (1977). Genotype-environment interaction and correlation in the analysis of human behavior. Psychological Bulletin 84, 309-322.

Raftery AE (1995). Sociological Methodology. Blackwell: Oxford, UK.

Rijsdijk FV, Sham PC, Sterne A, Purcell S, McGuffin P, Farmer A, Goldberg D, Mann A, Cherny SS, Webster M, Ball D, Eley TC, Plomin R (2001). Life events and depression in a community sample of siblings. Psychological Medicine 31, 401-410.

Schwartz G (1978). Estimating the dimension of a model. Annals of Statistics 6, 461-464.

Silberg J, Rutter M, Neale M, Eaves L (2001). Genetic moderation of environmental risk for depression and anxiety in adolescent girls. British Journal of Psychiatry 179, 116-121.

Spitz E, Moutier R, Reed T, Busnel MC, Marchaland C, Roubertoux PL, Carlier M (1996). Comparative diagnoses of twin zygosity by SSLP variant analysis, questionnaire, and dermatoglyphic analysis. Behavior Genetics 26, 55-63.

Thapar A, Harold G, McGuffin P (1998). Life events and depressive symptoms in childhood: shared genes or shared adversity? A research note. Journal of Child Psychology and Psychiatry 39, 1153-1158.

Van Os J, Jones PB (1999). Early risk factors and adult person-environment relationships in affective disorder. Psychological Medicine 29, 1055-1067.

Viken RJ, Rose RJ, Kaprio J, Koskenvuo M (1994). A developmental genetic analysis of adult personality: extraversion and neuroticism from 18 to 59 years of age. Journal of Personality and Social Psychology 66, 722-730.

Vinkhuyzen AA, van der Sluis S, de Geus EJ, Boomsma DI, Posthuma D (2010). Genetic influences on 'environmental' factors. Genes, Brain and Behavior 9, 276-287.

Wang JL, Schmitz N, Dewa C (2010). Socioeconomic status and the risk of major depression: the Canadian National Population Health Survey. Journal of Epidemiology and Community Health 64, 447-452.

Wichers M, Myin-Germeys I, Jacobs N, Peeters F, Kenis G, Derom C, Vlietinck R, Delespaul P, van Os J (2007). Genetic risk of depression and stress-induced negative affect in daily life. British Journal of Psychiatry 191, 218-223.

Wichers M, Schrijvers D, Geschwind N, Jacobs N, Myin-Germeys I, Thiery E, Derom C, Sabbe B, Peeters F, Delespaul P, van Os J (2009). Mechanisms of gene-environment interactions in depression: evidence that genes potentiate multiple sources of adversity. Psychological Medicine 39, 1077-1086. 